ARTICLE

Received 3 Dec 2013 | Accepted 6 May 2014 | Published 2 Jun $2014 \quad$ DOl: 10.1038/ncomms5049

\title{
Glass-glass transition during aging of a colloidal clay
}

Roberta Angelini ${ }^{1,2}$, Emanuela Zaccarelli ${ }^{2,3}$, Flavio Augusto de Melo Marques ${ }^{4}$, Michael Sztucki ${ }^{5}$, Andrei Fluerasu6 6 , Giancarlo Ruocco ${ }^{2,4} \&$ Barbara Ruzicka, ${ }^{1,2}$

Colloidal suspensions are characterized by a variety of microscopic interactions, which generate unconventional phase diagrams encompassing fluid, gel and glassy states and offer the possibility to study new phase and/or state transitions. Among these, glass-glass transitions are rare to be found, especially at ambient conditions. Here, through a combination of dilution experiments, X-ray photon correlation spectroscopy, small angle $X$-ray scattering, rheological measurements and Monte Carlo simulations, we provide evidence of a spontaneous glass-glass transition in a colloidal clay. Two different glassy states are distinguished with evolving waiting time: a first one, dominated by long-range screened Coulombic repulsion (Wigner glass) and a second one, stabilized by orientational attractions (Disconnected House of Cards glass), occurring after a much longer time. These findings may have implications for heterogeneously charged systems out-of-equilibrium and for applications where a fine control of the local order and/or long term stability of the amorphous materials are required.

\footnotetext{
${ }^{1}$ IPCF-CNR, UOS Roma, P.le Aldo Moro 2, I-00185 Roma, Italy. ${ }^{2}$ Dipartimento di Fisica, Sapienza Università di Roma, P.le Aldo Moro 2, I-00185 Roma, Italy. ${ }^{3}$ ISC-CNR, UOS Sapienza, P.le Aldo Moro 2, I-00185 Roma, Italy. ${ }^{4}$ Center for Life NanoScience, IIT@Sapienza, Istituto Italiano di Tecnologia, Viale Regina Elena 291, 00161 Roma, Italy. ${ }^{5}$ ESRF-The European Synchrotron, CS40220, 38043 Grenoble Cedex 9, France. ${ }^{6}$ Brookhaven National Laboratory, NSLS-II, Upton, New York 11973, USA. Correspondence and requests for materials should be addressed to R.A. (email: roberta.angelini@roma1.infn.it) or to B.R. (email: barbara.ruzicka@roma1.infn.it).
} 
$\mathrm{R}$ ecent advances in the study of soft materials have led in the last decades to a deeper knowledge of equilibrium and nonequilibrium states and to the discovery of new phases besides the ones commonly experienced in atomic or molecular systems. The tunability of colloidal effective interactions ${ }^{1}$ offers the possibility to observe unusual phase diagrams ${ }^{2}$ including reentrant $^{3,4}$ or empty liquid ${ }^{5,6}$ regimes, and multiple arrested states, such as gels ${ }^{7,8}$ and glassy states ${ }^{9,10}$. Of particular interest are glass-glass transitions ${ }^{3,4,11,12}$, which are quite rare to be found, especially at ambient conditions. Normally, these are triggered by a modification of the effective interparticle interactions that is obtained though a change of external parameters. For example, multiple glassy states were first experimentally observed in suspensions of hard-sphere-like particles such as sterically stabilized polymethylmethacrylate particles $^{3}$ or highly cross-linked microgel particles ${ }^{4}$, upon addition of non-adsorbing polymers. In these systems, a glassglass transition occurs from the well-known hard sphere glass ${ }^{9}$, due to caging in the absence of polymers, to a second glass, stabilized by the short-range attractive depletion interactions, on increase of polymer concentration ${ }^{13}$. The two glasses could then be distinguished by the different plateau values in their intermediate scattering functions $s^{3,4}$ and by their markedly difference rheological properties ${ }^{14}$.

In the wide panorama of soft matter systems, colloidal clays have gained increasing attention. In addition to their vast potential applications, their anisotropic shape and complex interactions considerably enrich their phase diagram ${ }^{6,15-17}$ and make them suitable candidates for self-assembly ${ }^{18}$. Among these, Laponite suspensions, widely used in cosmetics, oil industry, paints, have emerged as intriguing systems with peculiar aging dynamics and unexpected phase behaviour ${ }^{19-23}$. Since the pioneering work of Mourchid et al. ${ }^{24}$, a notable effort has been devoted to understand their very rich and debated state diagram. Different arrested states such as gels and glasses have been distinguished by varying clay and salt concentration ${ }^{21,23,25,26}$. The possible existence of two different types of glasses was first put forward by Tanaka and coworkers ${ }^{26}$ on increasing salt concentration: while a Wigner glass ${ }^{27-29}$ (dominated by electrostatic repulsion) was found at low ionic strength, an attractive glass (where platelets are connected through attractive bonds) was hypothesized at higher ionic strength. In this picture, at fixed clay concentration, a glass-glass transition should be observed on changing the ionic strength. Subsequent experiments by Jabbari et al. ${ }^{21}$ indeed found different dynamical behaviours on increasing salt concentration.

So far, the few glass-glass transitions reported have thus been observed on changing some key control parameter. At odds with these previous works, we work at fixed ionic strength, that is, in salt-free water conditions and at fixed clay concentration $C_{w}=3.0 \%$. We combine dilution experiments, X-Ray Photon Correlation Spectroscopy (XPCS), Small Angle X-ray Scattering (SAXS), rheological measurements and Monte Carlo (MC) simulations to provide evidence that during aging, without any change of external conditions, a spontaneous glass-glass transition takes place. Two different glassy states are distinguished with evolving waiting time. At first the system arrests, after a waiting time of the order of hours, into a Wigner glass dominated by long-range screened Coulombic repulsion in agreement with previous works ${ }^{26,28,30}$. However, at much longer waiting time, of the order of days, a second glass, previously unreported, is found. This is stabilized by orientational attractions between clay platelets and we propose to name it Disconnected House of Cards (DHOC) glass. The DHOC glass is different from the attractive glass proposed by Tanaka et al. ${ }^{26}$, since it takes place at low ionic strength where salt ions do not completely screen the negative charges of platelets giving rise to a net effective Coulombic repulsion among them. Therefore, while the nature of the attraction in both cases should be the same, originating from the presence of oppositely-signed charges on the platelet surface, platelets in the DHOC glass have to overcome a net repulsive barrier to get into contact hence remaining largely disconnected. Moreover, in the slow evolution of the microstructure in search for low-energy configurations, platelets rearrange into locally ordered structures with preferred T-shape in order to maximize the face-rim attraction, which takes place also at large distances. The reported glass-glass transition, originating from the competition between electrostatic repulsion and orientation-dependent attraction both acting at long range, is not a specific property of the investigated system but should be a generic feature of heterogeneously charged systems when driven out-of-equilibrium.

\section{Results}

Dilution experiments. Recently, dilution experiments have been used to identify ${ }^{30}$ that the arrested state at $2.0 \% \leq C_{w} \leq 3.0 \%$ is compatible with the formation of a Wigner glass ${ }^{27}$, that is, a glass stabilized by the long-range electrostatic repulsion between disconnected platelets. A similar dilution experiment on the Wigner glass exploring longer waiting times is reported here, with the discovery of a new and unexpected phenomenon. In Fig. 1a-1 we compare the evolution of the arrested state $(G)$, when deionized dyed water (L) is added (as in ref. 30) 1 day (a), 2 days (b), 3 days (c) and 4 days (d) after the arrest takes place. An initial identical situation for the four cases is observed (Fig. 1a-d). On addition of water, the arrested states in Fig. 1a,b start to fluidify with time (Fig. 1e,f) finally reaching a liquid state (Fig. 1i,j). On the contrary, the arrested states of the samples in Fig. 1c,d do not show any macroscopic change of their solid like state (Fig. 1g,h,k,l). The two different behaviours suggest that while the arrested states in panels a and $b$ (water added before 3 days) are dominated by repulsion-which develops immediately after the sample is prepared-and therefore are fluidified by the addition of water, the arrested states in panels $c$ and $d$ (water added after 3 days) have evolved into something different than a simple Wigner glass. We note that the time required for a sample at this concentration to age to the glassy state is of the order of hours; hence, this new transition occurs on a much longer timescale, when the system is already non-ergodic. Therefore an additional mechanism, probably due to attraction ${ }^{30}$, has intervened which does not allow the sample to become fluid any longer upon dilution. The results reported above suggest that a previously unreported transition occurs in glass Laponite samples at $C_{w}=3.0 \%$ around a critical time $t_{c} \approx 3$ days.

XPCS measurements. To further investigate this transition, we performed XPCS measurements at the ID10A beamline at the European Synchrotron Radiation Facility (ESRF). We measured the intensity autocorrelation functions of Laponite suspensions at $C_{w}=3.0 \%$ for rejuvenated samples as shown in Fig. $1 \mathrm{~m}$. The derived dynamic structure factor $f(Q, t)=\sqrt{g_{2}(Q, t)-1}$ is described by the Kohlrausch-Williams-Watts expression $f(Q, t) \sim \exp \left[-(t / \tau)^{\beta}\right]$ where $\tau$ is an 'effective' relaxation time, averaging the different relaxations that are simultaneously present in the system, while the exponent $\beta$ measures the distribution of such relaxations. While a stretched behaviour with $\beta<1$ has been reported for spontaneously aged samples at all waiting times, a surprising behaviour is found for the rejuvenated samples upon varying the rejuvenation time, $t_{R}$. Samples rejuvenated before a certain critical time $t_{c}$ show a stretched behaviour $(\beta<1)$ while samples rejuvenated after $t_{c}$ exhibit a 


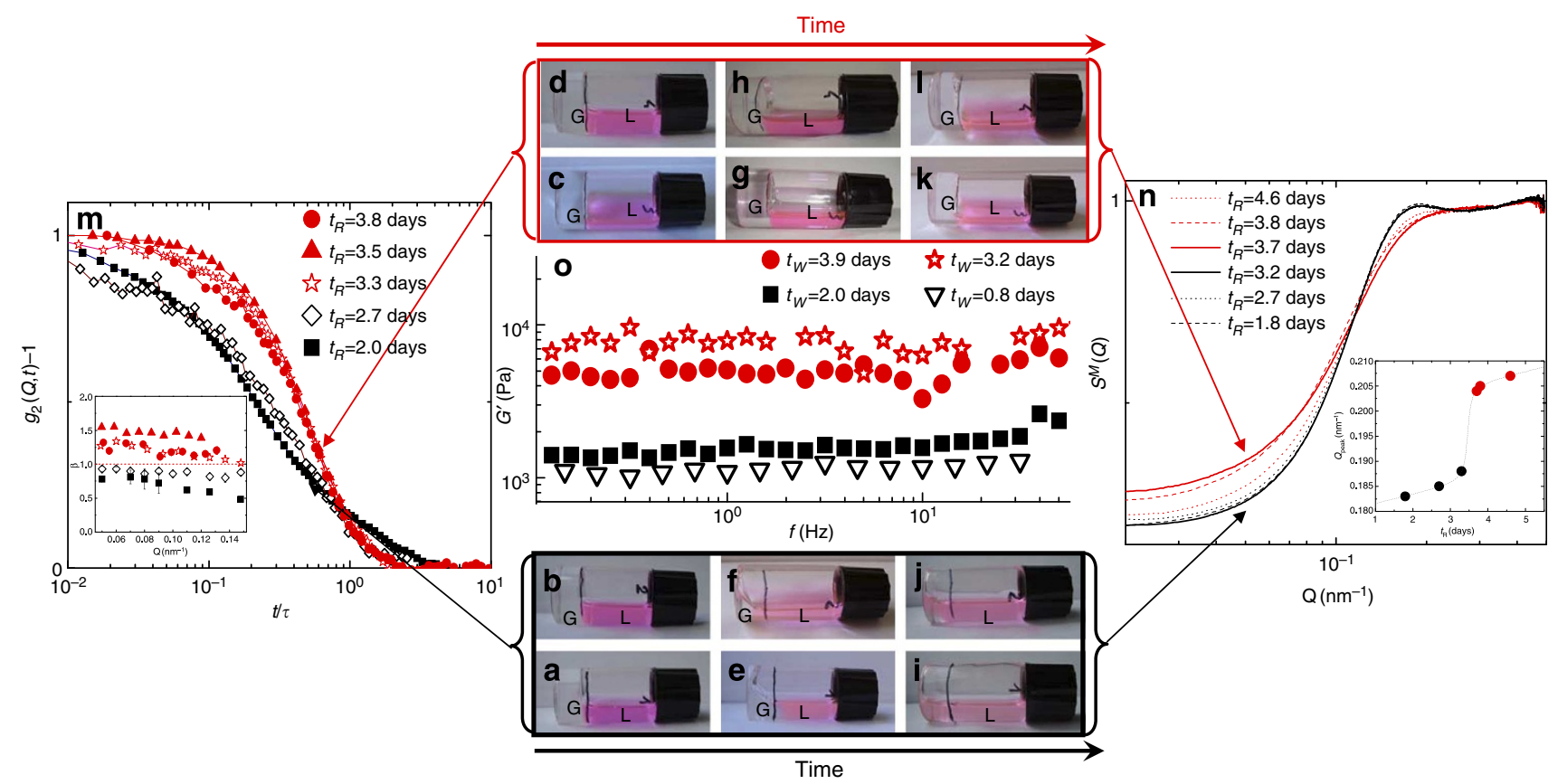

Figure 1 | Experimental behaviour of glassy Laponite suspensions at concentration $\mathbf{C}_{\boldsymbol{w}}=\mathbf{3 . 0} \%$. (a-I) Photographic time sequence of a dilution experiment for Laponite suspensions. The initially glassy sample (G) is added with the same volume of dyed water (L) 1 day (a), 2 days (b), 3 days (c) and 4 days (d) after arrest takes place. The samples $\mathbf{a}$ and $\mathbf{b}$ (water added before 3 days) start to fluidize (e,f) until the whole sample is completely liquid (i,j). The samples $\mathbf{c}$ and $\mathbf{d}$ (water added after 3 days) do not show any macroscopic change of their solid like state (g-k, h-I). (m) XPCS intensity autocorrelation functions for rejuvenated aqueous Laponite suspensions at different rejuvenation times $t_{R}: 2.0$ days (squares), 2.7 days (diamonds), 3.3 days (stars), 3.5 days (up triangles), 3.8 days (circles). In the inset, the $\beta$ exponent is shown as a function of Q. (n) Static structure factor for rejuvenated aqueous Laponite suspensions at different rejuvenation times $t_{R}: 1.8$ days (dashed black line), 2.7 days (dotted black line), 3.2 days (straight black line), 3.7 days (straight red line), 3.8 days (dashed red line), 4.6 days (dotted red line). In the inset, the peak position as a function of the rejuvenation time $t_{R}$ shows a transition between two different structures. (o) Frequency dependence of the elastic modulus $G^{\prime}$ at different waiting times $t_{w}: 0.8$ days (down triangles), 2.0 days (squares), 3.2 days (stars) and 3.9 days (circles).

compressed behaviour $(\beta>1)$ in agreement with a recent study ${ }^{31}$. This is shown in the inset of Fig. $1 \mathrm{~m}$ where the $\beta$ exponents are reported for samples rejuvenated before the critical time (squares, diamonds-black symbols; $t_{R}<t_{c}$ ) and for samples rejuvenated after the critical time (stars, triangles, circles-red symbols; $\left.t_{R}>t_{c}\right)$. We find this time to be $t_{c} \approx 3$ days for the studied clay concentration in agreement with the critical time $t_{c}$ identified through dilution measurements in Fig. 1a-l. These findings confirm the existence of two different behaviours below and above $t_{c}$ also in the dynamical properties of the system.

SAXS measurements. To complement these observations, we also investigate the structure of rejuvenated samples in order to assess whether the differences in the dynamics are also accompanied by changes in the structure. To this end, static structure factor measurements have been performed at the SAXS beamline ID02 at the ESRF. In Fig. 1n, the static structure factors $S^{M}(Q)$ of a rejuvenated Laponite suspension at concentration $C_{w}=3.0 \%$ are shown. Two different behaviours, separated by a change of $S^{M}$ $(Q)$, are found respectively for samples rejuvenated before and after $t_{c}$. Indeed, the $S^{M}(Q)$ of samples rejuvenated 1, 2 or 3 days after preparation (black curves) is similar to that previously reported for spontaneously aged samples ${ }^{30}$. On the other hand, the samples rejuvenated more than 3 days after preparation (red curves) show a change in $S^{M}(Q)$, which is characterized by a shift of the main peak to larger wave vectors and by a moderate increase of the low $\mathrm{Q}$ signal, that is, of the compressibility. In order to pinpoint the structural changes, we consider the position $Q_{\text {peak }}$ of the first maximum of $S^{M}(Q)\left(Q \sim 0.2 \mathrm{~nm}^{-1}\right)$ and report its evolution as a function of waiting time in the inset of Fig. 1n. The data show a jump that signs the transition between two different structures occurring at a time $t_{c} \approx 3$ days. However, we stress that even after the change at $t_{c}$ the average distance between platelets remains larger than the platelet size (that is, $\gtrsim 30 \mathrm{~nm}$ ) signalling that platelets are mainly not in contact.

Rheological measurements. To gain information on the macroscopic differences between the two glasses, we performed oscillatory rheological measurements at different times before and after $t_{c}$. For all the investigated times, the elastic modulus $G^{\prime}$ is always bigger (one order of magnitude) than the storage modulus $G^{\prime \prime}$, confirming that the system is in a solid like state. The results for the elastic modulus $G^{\prime}$ as a function of frequency in the arrested regime are shown in Fig. 10. At the reported waiting times, $t_{w}$ it displays an independent frequency behaviour over the probed frequency range. Interestingly a significant change of the elastic modulus is found: for times upto roughly 2 days (Wigner glass) $G^{\prime}$ is compatible with the long time behaviour reported in previous measurements ${ }^{28}\left(G^{\prime} \approx 10^{3} \mathrm{~Pa}\right)$; however, for times above 3 days, $G^{\prime}$ is roughly one order of magnitude higher $\left(G^{\prime}\right.$ $\approx 10^{4} \mathrm{~Pa}$ ). Such an increase was found in systems with depletion interactions when passing from a repulsive to an attractive glass $^{14,32}$. This result thus supports the existence of two glasses with significantly different elastic properties and suggests a crucial role of attraction for the occurrence of the spontaneous glassglass transition observed in Laponite suspensions. 
Discussion of experimental results. All of the above experimental observations, providing information on the microscopic structure (SAXS) and dynamics (XPCS) as well as on the macroscopic properties (dilution experiments and rheological measurements) of the glass, indicate the existence of two different behaviours of the arrested state before and after 3 days. Indeed we have observed a spontaneous transition between two distinct dynamically arrested states, without an intervening intermediate (ergodic) state, that is, a glass-glass transition occurring during the aging process. This suggests a non-trivial interplay between repulsive and attractive interactions at work in Laponite suspensions. Dilution experiments suggest a change in the nature of the arrested state with waiting time: a stabilization of the glass through attractive interaction does not permit to the glass to fluidify any longer upon addition of water. This attractive interaction between oppositely charged faces and rims of the platelets, was already found to play a central role in the formation of a gel network at lower concentrations on very long timescales ${ }^{6}$. We thus conjecture that two separate timescales control the role of the different interactions: while repulsion is felt almost immediately after samples are prepared, attraction, due to its anisotropic nature and to the presence of an effective repulsive barrier, becomes effective on a much longer timescale ${ }^{30}$. Therefore, the novel transition taking place even at high concentrations $\left(C_{w}=3.0 \%\right)$ on a retarded timescale alerts us on the possible development of attraction even in the Wigner glass regime at long timescales. The rheological measurements confirm that the transition occurs between a repulsive-dominated and an attractive-dominated glass.

Simulations. To gain a microscopic understanding of this transition and to clarify the nature of the attraction, we have carried out MC simulations of a simple model of Laponite platelets which combines both repulsive and attractive ingredients. Platelets are represented as rigid disks as in refs 6,33, interacting via an effective potential $V_{\text {tot }}$, which is the sum of different contributions.

First, we consider a screened electrostatic repulsion

$$
V_{\text {rep }}=A \frac{\exp (-r / \lambda)}{r}
$$

where $\lambda$ is the Debye length and $A$ the repulsion strength that depends quadratically on the excess effective charge $Z^{\text {ex }}$. This (negative) excess charge results from the balance between negative and positive charges inhomogeneously distributed on the platelets. The Debye length and excess effective charge are fixed as described in the Methods and Supplementary Note 1 (see also Supplementary Fig. 1).

Secondly, to model the attractive interaction between positive and negative charges, located respectively on the rim and on the face of each platelet, we complement the short-range patchy term $V_{\text {patchy }}$ used in ref. 6 (see Methods and Supplementary Fig. 2, and Supplementary Note 2) with a long-range orientational term $V_{\text {lr }}$ of quadrupolar nature ${ }^{34}$. The patchy potential amounts to decorate each platelet with five sites, three located symmetrically on the rim and two on the opposite faces of the platelet. Site-site attraction acts only between rim and face sites and is modelled as a short-ranged square well of depth $\epsilon$. The long-range term is defined as,

$$
V_{\mathrm{lr}}=-\epsilon\left(\frac{r_{0}}{r}\right)^{5}\left[1-\cos ^{2}(\theta)\right]
$$

where $\theta$ is the angle formed by the normal of the two interacting platelets and $r_{0}$ is the contact distance for the T-bond configuration (see Fig. 2a). The value of attraction at contact $(\epsilon)$ is chosen to match the patchy attraction term so that
T-bonded configurations are those of minimum energy (see Methods). In essence, platelets interact with $V_{\text {tot }}=V_{\text {rep }}+V_{\text {att }}$ (see Fig. $2 \mathrm{~b})$, where the attraction $\left(V_{\mathrm{att}}=V_{\text {patchy }}+V_{\mathrm{lr}}\right)$ plays the role of a higher order term in the multipole expansion of the charge distribution on each Laponite platelet.

In Fig. 2c, we report the static structure factors $S^{\operatorname{sim}}(Q)$ between the platelets centres of mass calculated within MC simulations for $V_{\text {tot }}$ at the experimental number density as a function of reduced temperature, $T^{\star}=k_{B} T / \epsilon$ (where $T$ is the temperature and $k_{B}$ the Boltzmann constant). The decrease of temperature produces an effect on $S^{\operatorname{sim}}(Q)$ that is qualitatively similar to the behaviour observed in experiments (Fig. 1n). Indeed previous works $^{35}$ have suggested that increasing waiting time can be regarded as a decrease of $T^{*}$, mimicking aging evolution. We see that, in simulations as in experiments, the structure factor peak moves to larger values of $Q$ and the low $Q$ signal increases upon decreasing $T^{\star}$.

We stress here that our model is still a very crude one with respect to the real Laponite-Laponite interactions. Hence, this should be used as a guidance for physical insights of the main mechanisms driving the experimental behaviour. Indeed, without relying on the particular choice of Equation 2 the same qualitative results can be obtained by using the quadrupolar model of Dijkstra et al. ${ }^{34}$ in addition to $V_{\text {rep }}$ (Supplementary Fig. 3 and Supplementary Note 3 ). Surely more sophisticated efforts ${ }^{23,36}$ will be needed in the future to reproduce the experimental data at a more quantitative level. Nonetheless, our simple model made by an electrostatic screened repulsion plus an orientational longrange attraction allows us to interpret at microscopic level the observed glass-glass transition. At the studied concentration, a few hours after sample preparation, platelets feel essentially a strong electrostatic repulsion due to the net excess negative charge on their surface (Wigner glass). Despite the system being arrested, some small rattling and, most importantly, adjustments of the relative orientations are possible, so that after some time $t_{c}$ the system, still within the non-ergodic state, rearranges acquiring a T-bond driven orientational order. This is stabilized by a longrange orientational attraction, which does not allow the system to melt any longer upon dilution. To visualize the local organization of platelets we show in Fig. 2d,e typical simulation snapshots at two different $T^{*}$, where we have coloured platelets according to the relative orientation to the central one. A large set of mutually orthogonal platelets is present at low $T^{\star}$ (see Fig. 2e), which extends beyond first neighbours. However, not all neighbour platelets are orthogonal to each other, since there is a coexistence of several patterns of mutually orthogonal platelets (a sort of antinematic regions), thereby generating the glassiness of the samples and the absence of nematic order. It is important to stress that in the simulations we do not find evidence of large clusters of platelets: indeed only about $10 \%$ of them are engaged in contact (patchy) bonds. Platelets thus remain largely disconnected as in the Wigner glass case but develop a longrange order preferentially along orthogonal directions with respect to their nearest neighbours (Supplementary Fig. 4 and Supplementary Note 4). This is in agreement with the position of the experimental $S^{M}(Q)$ peak which saturates at long times to around $\mathrm{Q} \sim 0.2 \mathrm{~nm}^{-1}$, a value still much smaller than that corresponding to the house of cards connected structure ${ }^{23}$. These findings allow us to identify the novel type of orientational glass observed at large waiting times as a Disconnected House Of Card glass.

\section{Discussion}

In the present work, we have reported evidence of a glass-glass transition: a translational Wigner glass makes a spontaneous 
a

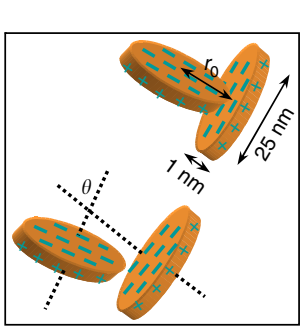

b

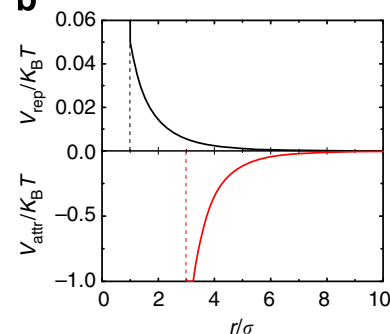

c

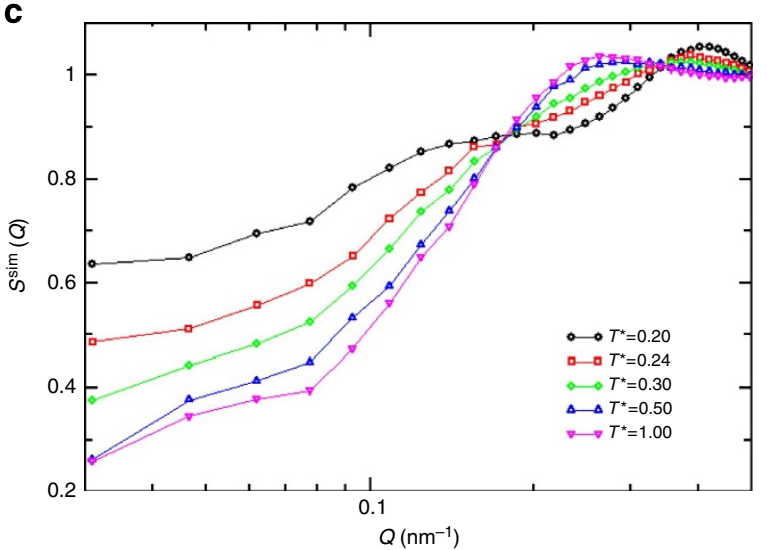

$Q\left(\mathrm{~nm}^{-1}\right)$

d

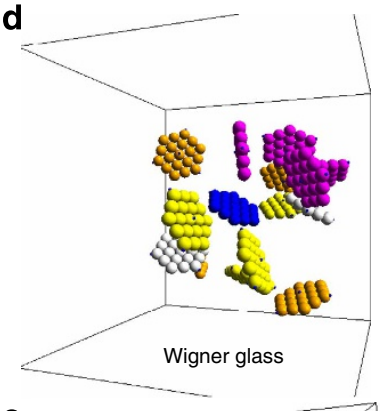

e

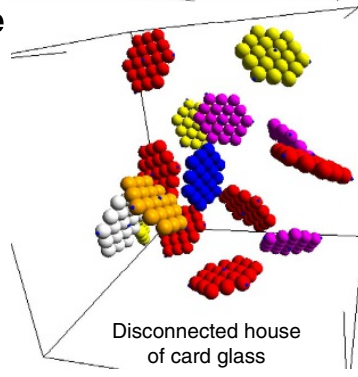

$0-30^{\circ}$

Figure 2 | Simulated static structure factor of Laponite suspensions and snapshots. (a) Cartoon representing two interacting Laponite platelets. (b) Interaction potential between Laponite particles: top Yukawa repulsion, bottom attraction for orthogonal platelets $\left(\theta=90^{\circ}\right)$. (c) Simulated static structure factor of platelets interacting via electrostatic repulsion and a long-range orientational attraction at fixed $C_{w}=3.0 \%$ on varying attraction strength. This mimics the effect of time taking place in the aging of Laponite suspensions, producing a qualitative similar behaviour for the structure factors as in the experiments. (d,e) Three-dimensional snapshots of MC simulations of a shell of platelets of width $10 \sigma$ around a central one (blue platelet) to highlight orientational order that is measured by the relative angle $\theta$ according to the colour bar. Red platelets are almost orthogonal to the central one, while white ones are almost parallel. (d) Snapshot at $T^{\star}=1.0$ : the orientations are completely random and orthogonal platelets can be found or not (as in the example). (e) Snapshot at $T^{\star}=0.24$ : a relative order in the orientation develops which evolves even to the second neighbour shell, while the number of nearby platelets with different orientations decreases. Note that the box shown is only a part of the total box and that the number density of platelets is the same in the two snapshots.

transition with waiting time in a new partially orientationally ordered DHOC glass. We have proposed that the latter is stabilized by orientational attractions between clay platelets arising from their heterogeneous charge distribution. This hypothesis is confirmed by results of computer simulations of disks interacting both with competing repulsion and orientationdependent attraction. This complex structure with mutually orthogonal platelets within a disordered matrix indicates a partial orientational order. With respect to the attractive glass previously identified at large ionic strength ${ }^{26}$, the DHOC glass builds from a previous Wigner glass and maintains its microstructure with platelets relatively far from each other. Indeed, $S(Q)$ does not show the typical feature of an attractive system, for example, a growth at low wave vectors. Thus, the average distance between platelets remains largely dictated by the long-range repulsion. On the contrary, we expect that the addition of salt, necessary to obtain an attractive glass in ref. 26, would also affect this structure, creating a connected network of platelets. Therefore, in the DHOC glass, platelets cannot modify significantly their interparticle distance but can adjust their mutual orientations in order to minimize the energy through preferred T-shaped configurations. Hence, while the two attractive glasses reported here and proposed in ref. 26 share the same mechanism of attraction, due to the face-rim electrostatic attraction, their characteristics such as structure and rheological properties should be rather different. In this respect, it will be important to assess how the glass-glass transition is affected by adding salt and by changing clay concentration. It would be very interesting in the future to systematically investigate the transition and the conditions by which it may also be speeded up and/or disappear. Another way that seems promising to characterize the transition is the study of the role of rotational and translational dynamics. Recent studies $^{37,38}$ have shown a decoupling between the two degrees of freedom in the early glass, which could be important in the subsequent evolution towards the partially ordered glass.

The occurrence of the DHOC glass opens also another set of questions regarding the connection with previously reported nematic order $22,24,39-41$. Such nematic states should now be revisited to identify whether there could be more than one nematic axis or whether the formation of a nematic state completely destroys the $T$-shaped configurations.

Finally, we expect that the found glass-glass transition could be a generic feature of heterogeneously charged or patterned ${ }^{18}$ systems with competing attractive-repulsive electrostatic interactions when driven out-of-equilibrium towards an arrested state. The practical importance of our findings for technological applications could be twofold. On one hand, the knowledge of the microstructure is necessary when a fine control of the local order at the microscopic scale is relevant for the mesoscopic scale, as for example in transport processes through an amorphous matrix. On the other hand, the observation of a glass that spontaneously transforms in time without external change of any control parameter (clay concentration, salt concentration, $\mathrm{pH}$, temperature and so on) needs to be taken into account for assessing the long term stability of the amorphous materials. 


\section{Methods}

Laponite sample preparation. Laponite RD suspensions were prepared and sealed in a glove box under $\mathrm{N}_{2}$ flux to avoid samples degradation ${ }^{23}$. The powder, manufactured by Rockwood Ltd, was firstly dried in an oven at $T=400{ }^{\circ} \mathrm{C}$ for $4 \mathrm{~h}$ and it was then dispersed in pure deionized water at salt concentration $C_{s} \simeq 10^{-4} \mathrm{M}$, stirred vigorously for $30 \mathrm{~min}$ and filtered soon after through $0.45 \mu \mathrm{m}$ pore size Millipore filters. The origin of the waiting time $\left(t_{w}=0\right)$ is the time of filtration of the suspension. The same identical protocol has been strictly followed for the preparation of each sample, fundamental condition to obtain reliable and reproducible results ${ }^{23}$. All our samples after a few minutes reach a constant value of $p H \simeq 10$, as measured in ${ }^{42,43}$. At this $\mathrm{pH}$ the rim charges are positive ${ }^{44}$. Indirect evidence of the existence of positive rim charges is the occurrence of phase separation in the absence of salt for low concentration samples ${ }^{6}$. The rejuvenated sample has been obtained by refluidifing a glassy sample by applying a shear with a syringe. The rejuvenation time $t_{R}$ is the time elapsed between sample preparation and application of the shear. Samples were placed and sealed in $2.5 \mathrm{~cm}$ diameter glass bottles for dilution experiments, in $2 \mathrm{~mm}$ diameter capillaries for both XPCS and SAXS measurements and on the plate of the rheometer for rheological measurements.

Dilution. For the dilution experiment, a solution of coloured water with Rhodamine $\mathrm{B}$ at a concentration $10^{-6} \mathrm{M}$ with the same volume as that of the Laponite solution is added to the arrested (colourless) sample at clay concentration, $C_{w}=3.0 \%$. We have verified that the addition of the dye does not affect the results performing the same experiment in absence of colourant ${ }^{30}$.

XPCS. XPCS measurements were performed at the ID10A beamline at the ESRF in Grenoble, France using an incident partially coherent X-ray beam with energy fixed at $8 \mathrm{keV}$. A series of scattering images were recorded by a charged coupled device and the ensemble averaged intensity autocorrelation function

$g_{2}(Q, t)=\frac{\left\langle\left\langle I\left(Q, t_{0}\right) I\left(Q, t_{0}+t\right)\right\rangle_{p}\right\rangle}{\left\langle\left\langle I\left(Q, t_{0}\right)\right\rangle_{p}\right\rangle^{2}}$, where $\langle\ldots\rangle_{p}$ is the ensemble average over the detector pixels mapping onto a single $\mathrm{Q}$ value and $\langle\ldots\rangle$ is the temporal average over $t_{0}$, was calculated by using a standard multiple $\tau$ algorithm ${ }^{45}$.

SAXS. SAXS measurements were performed at the High Brilliance beam line (ID02) at ESRF using a $10 \mathrm{~m}$ pinhole SAXS instrument. The incident X-ray energy was fixed at $12.6 \mathrm{keV}$. The form factor $F(Q)$ was measured using a flow-through capillary cell. SAXS data were normalized and the scattering background of water was subtracted. The measured structure factor has been obtained as $S^{M}(Q)=I(Q) / F(Q)$. The peak positions shown in the inset of Fig. 1n have been determined by performing a gaussian fit in the range $0.14-0.20 \mathrm{~nm}^{-1}$ for the samples $1.8,2.7$ and 3.2 days and in the range $0.15-0.22 \mathrm{~nm}^{-1}$ for the samples 3.7, 3.8 and 4.6 days.

Rheological measurements. Oscillatory rheological measurements were performed with a MCR302 Anton Paar rheometer in a plate-plate geometry in controlled normal force configuration and with shear stress $\sigma=0.5 \mathrm{~Pa}$.

Simulations. Each platelet is modelled as a hard rigid disc composed of 19 sites on a hexagonal mesh, inspired by ref. 33. Each site is a hard-sphere of diameter $\sigma$ so that overlap between different sites is forbidden. A comparison with Laponite fixes $\sigma=5 \mathrm{~nm}$. We have simulated 200 platelets in a cubic box at fixed number density $\left(2.4 \times 10^{-5} \mathrm{~nm}^{-3}\right)$, whose value coincides with the experimental one.

The sites on the disk interact via a Yukawa potential (Eq. 1) whose strength is $A=\left(Z^{e x} e\right)^{2} / 19 \epsilon_{0} \epsilon_{R}$, where $Z^{e x}$ is the site excess (average) negative charge on the platelet surface, $e$ the elementary charge and $\epsilon_{0}, \epsilon_{R}$ the dielectric constants respectively of vacuum and of water. The values of the Debye length $\lambda=9 \mathrm{~nm}$ and $Z^{e x}=15 e$ are chosen by comparison with the experimental structure factors in the Wigner glass regime (Supplementary Fig. 1 and Supplementary Note 1) and then kept fixed throughout, as the experiments are carried out at fixed salt concentration. An attractive patchy contribution is added by decorating each platelet with five sites, three located symmetrically on the rim and two on the two opposite faces of the central hard sphere. Site-site attraction acts only between rim and face sites and is modelled as a square well term, with range $\sigma_{\mathrm{p}}=0.1197 \sigma$ and depth $\epsilon=1$. The addition of a simple patchy attraction is not sufficient to reproduce the behaviour of the experimental structure factors, as shown in Supplementary Fig. 2, and discussed in the Supplementary Note 2.

The addition of the long-range orientational attraction is made in such a way that it extends the patchy contribution to longer distances. Hence, the orientational strength is chosen to match the patchy attraction at contact, corresponding to $r_{0}=3 \sigma+2 \sigma_{\mathrm{p}}$, when platelets are orthogonal (that is, $\cos (\theta)=0$ ) (see Fig. 2a). In order to ensure that T-bonded configurations are those of minimum energy, longrange orientational attraction only takes place when $r>r_{0}$ (that is, outside of the patchy well). The absence of attraction for smaller $r$ was also adopted by Dijkstra et al. ${ }^{34}$ to avoid an unphysical contribution of overlapped platelets. The latter can only be included in a proper way once discrete opposite charges are explicitly taken into account ${ }^{36}$.

\section{References}

1. Likos, C. N. Effective interactions in soft condensed matter physics. Phys. Rep. 348, 267-439 (2001).

2. Anderson, V. J. \& Lekkerkerker, H. N. W. Insights into phase transition kinetics from colloid science. Nature 416, 811-815 (2002).

3. Pham, K. N. et al. Multiple glassy states in a simple model system. Science 296, 104-106 (2002).

4. Eckert, T. \& Bartsch, E. Re-entrant glass transition in a colloid-polymer mixture with depletion attractions. Phys. Rev. Lett. 89, 125701 (2002).

5. Bianchi, E., Largo, J., Tartaglia, P., Zaccarelli, E. \& Sciortino, F. Phase diagram of patchy colloids: towards empty liquids. Phys. Rev. Lett. 97, 168301 (2006).

6. Ruzicka, B. et al. Observation of empty liquids and equilibrium gels in a colloidal clay. Nat. Mater. 10, 56-60 (2011).

7. Lu, P. J. et al. Gelation of particles with short-range attraction. Nature 453, 499-503 (2008).

8. Royall, C. P., Williams, S. R., Ohtsuka, T. \& Tanaka, H. Direct observation of a local structural mechanism for dynamical arrest. Nat. Mater. 7, 556-561 (2008).

9. Pusey, P. N. \& van Megen, W. Phase behaviour of concentrated suspensions of nearly hard colloidal spheres. Nature 320, 340-342 (1986).

10. Imhof, A. \& Dhont, J. K. G. Experimental phase diagram of a binary colloidal hard-sphere mixture with a large size ratio. Phys. Rev. Lett. 75, 1662-1665 (1995).

11. Chen, S.-H., Chen, W.-R. \& Mallamace, F. The glass-to glass transitions and its end point in copolymer micellar system. Science 300, 619-622 (2003).

12. Mayer, C. et al. Asymmetric caging in soft colloidal mixtures. Nat. Mater. 7, 780-784 (2008).

13. Sciortino, F. Disordered materials: one liquid, two glasses. Nat. Mater. 1, 145-168 (2002).

14. Pham, K. N. et al. Yielding behavior of repulsion-and attraction-dominated colloidal glasses. J. Rheol. 52, 649-676 (2008).

15. Michot, L. J. et al. Liquid-crystalline aqueous clay suspensions. PNAS 103, 16101-16104 (2006).

16. Shalkevich, A., Stradner, A., Bhat, S. K., Muller, F. \& Schurtenberger, P. Cluster, glass, and gel formation and viscoelastic phase separation in aqueous clay suspensions. Langmuir 23, 3570-3580 (2007).

17. Mourad, M. C. D. et al. Sol-gel transitions and liquid crystal phase transitions in concentrated aqueous suspensions of colloidal gibbsite platelets. J. Phys. Chem. B 113, 11604-11613 (2009).

18. Glotzer, S. C. \& Solomon, M. J. Anisotropy of building blocks and their assembly into complex structures. Nat. Mater. 6, 557-562 (2007).

19. Bandyopadhyay, R. et al. Evolution of particle-scale dynamics in an aging clay suspension. Phys. Rev. Lett. 93, 228302 (2004).

20. Schosseler, F., Kaloun, S., Skouri, M. \& Munch, J. P. Diagram of the aging dynamics in laponite suspensions at low ionic strength. Phys. Rev. E 73, 021401 (2006).

21. Jabbari-Farouji, S., Tanaka, H., Wegdam, G. H. \& Bonn, D. Multiple nonergodic disordered states in Laponite suspensions: a phase diagram. Phys. Rev. E 78, 061402 (2008).

22. Shahin, A. \& Joshi, Y. M. Irreversible aging dynamics and generic phase behavior of aqueous suspensions of laponite. Langmuir. 26, 4219-4225 (2010)

23. Ruzicka, B. \& Zaccarelli, E. A fresh look at the laponite phase diagram. Soft Matter 7, 1268-1286 (2011).

24. Mourchid, A., Lecolier, E., Van Damme, H. \& Levitz, P. On viscolelastic, birefringent, and swelling properties of laponite clay suspensions: revisited phase diagram. Langmuir 14, 4718-4723 (1998).

25. Mongondry, P., Tassin, J. F. \& Nicolai, T. Revised state diagram of laponite dispersions. J. Colloid Interface Sci. 283, 397-405 (2005).

26. Tanaka, H., Meunier, J. \& Bonn, D. Nonergodic states of charged colloidal suspensions: repulsive and attractive glasses and gels. Phys. Rev. E 69, 031404 (2004).

27. Lindsay, H. M. \& Chaikin, P. M. Elastic properties of colloidal crystals and glasses. J. Chem. Phys. 76, 3774-3781 (1982).

28. Bonn, D., Tanaka, H., Wegdam, G., Kellay, H. \& Meunier, J. Aging of a colloidal 'wigner' glass. Europhys. Lett. 45, 52-57 (1999).

29. Levitz, P., Lecolier, E., Mourchid, A., Delville, A. \& Lyonnard, S. Liquid-solid transition of laponite suspensions at very low ionic strenght: long-range electrostatic stabilitation of anisotropic colloids. Europhys. Lett. 49, 672-677 (2000).

30. Ruzicka, B. et al. Competing interactions in arrested states of colloidal clays. Phys. Rev. Lett. 104, 085701 (2010).

31. Angelini, R. et al. Dichotomic aging behavior in a colloidal glass. Soft Matter 9, 10955-10959 (2013).

32. Truzzolillo, D. et al. Glassy states in asymmetric mixtures of soft and hard colloids. Phys. Rev. Lett. 111, 208301 (2013).

33. Kutter, S., Hansen, J., Sprik, M. \& Boek, E. Structure and phase behavior of a model clay dispersion: A molecular-dynamics investigation. J. Chem. Phys. 112, 311-322 (2000). 
34. Dijkstra, M., Hansen, J. \& Madden, P. A. Statistical model for the structure and gelation of smectite clay suspensions. Phys. Rev. E 55, 3044-3053 (1997).

35. Sciortino, F. et al. A parameter-free description of the kinetics of formation of loop-less branched structures and gels. Soft Matter 5, 2571-2575 (2009)

36. Delhorme, M., Jönsson, B. \& Labbez, C. Monte carlo simulations of a clay inspired model suspension: the role of rim charge. Soft Matter 8, 9691-9704 (2012).

37. Jabbari-Farouji, S., Wegdam, G. H. \& Bonn, D. Aging of rotational diffusion in colloidal gels and glasses. Phys. Rev. E 86, 041401 (2012).

38. Jabbari-Farouji, S., Wegdam, G. H. \& Bonn, D. Dynamical heterogeneity in aging colloidal glasses of Laponite. Soft Matter 8, 5507-5512 (2012).

39. Gabriel, J.-C. P., Sanchez, C. \& Davidson, P. Observation of nematic liquid-crystal textures in aqueous gels of smectite clays. J. Phys. Chem. 100, 11139-11143 (1996)

40. Lemaire, B. J., Panine, P., Gabriel, J. C. P. \& Davidson, P. The measurement by SAXS of the nematic order parameter of laponite gels. Europhys. Lett. 59, 55-61 (2002).

41. Hansen, E. L., Jabbari-Farouji, H., Mauroy, T. S., Plivelicand, D. \& Bonn, J. O. Fossum, orientational order in a glass of charged platelets with a concentration gradient. Soft Matter 9, 9999-10004 (2013).

42. Cummins, H. Z. Liquid, glass, gel: the phases of colloidal laponite. J. Non-Cryst. Solids 353, 3892-3905 (2007).

43. Martin, C. et al. Dissociation of thixotropic clay gels. Phys. Rev. E 66, 021401 (2002).

44. Tawari, S. L., Koch, D. L. \& Cohen, C. Electrical double-layer effects on the brownian diffusivity and aggregation rate of laponite clay particles. J. Colloid Interface Sci. 240, 54-66 (2001).
45. Madsen, A., Leheny, R., Guo, H., Sprung, M. \& Czakkel, O. Beyond simple exponential correlation functions and equilibrium dynamics in $\mathrm{x}$-ray photon correlation spectroscopy. New J. Phys. 12, 055001 (2010).

\section{Acknowledgements}

We acknowledge the European Synchrotron Radiation Facility for provision of beamtime and G. De Bellis from CNIS-Sapienza for assistance during rheological measurements and F. Sciortino for comments on the manuscript. R.A. and E.Z. acknowledge support from MIUR-PRIN. E.Z. acknowledges support from MIUR-FIRB ANISOFT (RBFR125H0M),

\section{Author contributions}

R.A. and B.R. carried out experiments, analysed the data, discussed the results and wrote the manuscript. E.Z. did the modelling and numerical simulations, discussed the results and wrote the manuscript. F.A.d.M.M. prepared the samples and carried out experiments. M.S. and A.F. gave technical support respectively for SAXS and XPCS experiments. G.R. discussed the results and provided suggestions and comments.

\section{Additional information}

Supplementary Information accompanies this paper at http://www.nature.com/ naturecommunications

Competing financial interests: The authors declare no competing financial interest.

Reprints and permission information is available online at http://npg.nature.com/ reprintsandpermissions/

How to cite this article: Angelini, R. et al. Glass-glass transition during aging of a colloidal clay. Nat. Commun. 5:4049 doi: 10.1038/ncomms5049 (2014). 